\section{Laparoscopic Cholecystectomy: A Case of Postoperative Hemorrhage Successfully Treated by Laparoscopic Reintervention}

We report here on a case of postoperative hemorrhage after laparoscopic cholecystectomy, which was successfully treated by laparoscopic reintervention.

A 57-year-old man was diagnosed with acute cholecystitis. Three weeks after the acute inflammation had subsided laparoscopic cholecystectomy was carried out, following techniques previously described $(1-3)$. Pulsatile bleeding was seen from the thickened stump of the cystic duct, and this was stopped by double-clipping the stump. The cystic artery was also double-clipped, and the gallbladder was removed from the fossa using electrocoagulation. The operation was completed after it was confirmed that bleeding had ceased.

Six hours postoperatively, the bandage was stained with fresh blood from the film drains. The hemoglobin level had decreased to 7.1 $\mathrm{g} / \mathrm{dl}$ at 36 hours postoperatively (Figure 1 ). It was decided to carry out a repeat laparoscopic intervention as an emergency procedure in the operating room, with a diagnosis of postoperative intra-abdominal hemorrhage. A large quantity of coagulated blood was found within the abdominal cavity, mainly in the caudal to lateral region of the right lobe of the liver. To locate the origin of the bleeding in the area of the liver bed and from the stumps of the cystic duct or artery, the coagulated blood was scrupulously and perseveringly removed using an irrigating aspirator $5 \mathrm{~mm}$ in diameter. After this, it was possible to confirm without much difficulty that the liver bed and the stump of the cystic artery were intact. While the coagulated blood was being removed around the stump of the divided cystic duct, a pulsatile hemorrhage was found (Figure 2). There was a small artery running in parallel with the cystic duct along the line of the open side of the clips previously applied during the first operation. While the stump of the divided cystic duct was held up with forceps, the cystic duct was doubly ligated using an Endoloop, together with the small bleeding artery. The postoperative course was uneventful, and the patient was discharged on the eighth postoperative day without having received any blood transfusion.

After the present case, double ligation of the cystic duct using an Endoloop has been performed in all cases in which pulsatile bleeding was found at the stump of the divided cystic duct. No similar cases have since occurred in a series of 900 operations. An abdominal drain was inserted in all patients undergoing laparoscopic cholecystectomy as an information drain or insurance drain to provide immediate information about any complications of postoperative hemorrhage or bile leakage.

In conclusion, it is advised that a drain should be inserted in all patients undergoing laparoscopic cholecystectomy, as this may allow laparoscopic reintervention instead of open laparotomy (4) in case of postoperative accidents. Although the incidence of such accidents is likely to be extremely low, early information provided by an insurance drain would effectively prevent patients from developing serious postoperative complications.

\section{K. Ido', C. Kawamoto', K. Kimura', T. Suzuki ${ }^{l}$, Y, Taniguchi', \\ N. Isoda', M. Kumagai \\ ' Dept. of Endoscopy, Jichi Medical School, Yakushiji, \\ Tochigi-ken, Japan;}

${ }^{2}$ Dept. of Surgery, Imaichi Hospital, Imaichi, Tochigi-ken, Japan

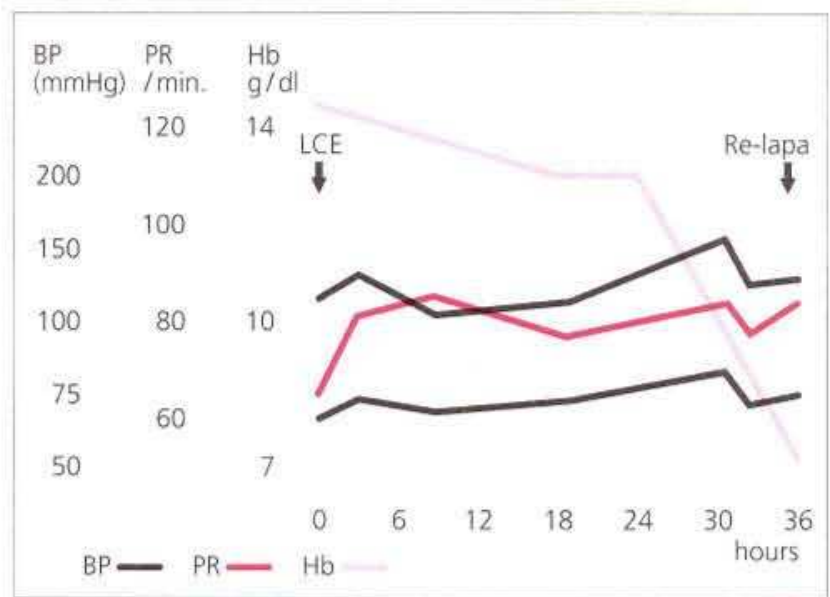

Figure 1: The clinical course of the present case, from cholecystectomy to the laparoscopic reintervention.

$\mathrm{BP}$ Blood pressure

$\mathrm{Hb}$ Hemoglobin

LCE Laparoscopic cholecystectomy

PR Pulse rate

Re-lapa.Laparoscopic reintervention

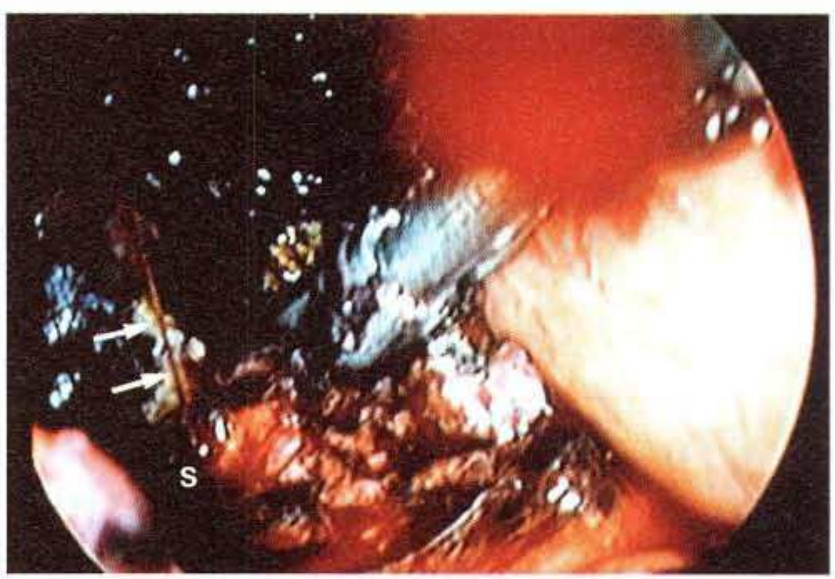

Figure 2: Pulsatile bleeding (arrows) was starting again as coagulated blood was removed from around the cystic duct.

5 The stump of the cystic duct

\section{References}

1. Kimura K. Ido K. Taniguchi Y, et al. Prospective study of cholecystectomy in two hundred and fifty patients. Endoscopy 1992; 24:750-3

2. Ido K, Kimura K, Kawamoto C, et al. Laparoscopic transcystic cholangioscopy for treatment of common bile duct stones: preliminary experience. Endoscopy 1992; 24:749-52.

3. Taniguchi $\mathrm{Y}$, Ido K, Kimura K, et al. Introduction of a "safety zone" for the safety of laparoscopic cholecystectomy. Am J Gastroenterol 1993; 88: 1258-61.

4. Voyles CR, Petro AB, Meena AL, et al. A practical approach to laparoscopic cholecystectomy, Am J Surg 1991; 161: 365-70.

Corresponding Author

K. Kimura, M.D., Dept. of Endoscopy

Jichi Medical School, Yakushiji

Tochigi-ken 329-04, Japan

Fax: $+81-285-448297$ 